\title{
Devoicing of Japanese vowels by Taiwanese learners of Japanese
}

\author{
J. Kevin Varden\& Tsutomu Sato \\ (varden@meijigakuin.ac.jp, tsato@meijigakuin.ac.jp) \\ Dept. of English, Meiji Gakuin University
}

\begin{abstract}
Three Taiwanese speakers studying in Japan were tested for application of the Japanese rule of High Vowel Devoicing. Three native speakers of Japanese served as controls. Five repetitions each of tokens containing two possible devoicing sites were collected from each participant at speaker-determined slow and fast speeds.
\end{abstract}

Of the Taiwanese participants, only one showed any significant speech rate effect $(t=1.88, p<.05$ for the first vowel of the tokens; $t=2.29, p<.025$ for both vowels combined). One other showed no speech rate effects whatsoever, instead inconsistently devoicing some tokens, while the third did not devoice any vowels within the tokens, instead only devoicing a small number of sentence-final vowels.

It appears that each of the three learners has begun to devoice vowels according to different processes-for one speaker, a speech rate dependent rule; for another, a speech rate insensitive rule whose environment is not yet solidified; for the third, as a rule sporadically applied to the end of a sentence.

Most surprisingly, native speakers also showed a lack of significant speech rate effects. This would imply that High Vowel Devoicing is losing its status as a fast speech rule in the Tokyo dialect. Further, larger studies will show if this indication is correct.

\section{INTRODUCTION}

Much has been written about the acquisition of phonemic categories for sounds acquired in second languages [1]. However, little work has been done on the acquisition of phonological rules, largely because the lack of consensus surrounding phonological issues in first language acquisition is amplified within the bilingual, second, or foreign language learner [2]. This paper reports on an attempt to study the acquisition of a phonological rule by language learners, the acquisition of the rule of High Vowel Devoicing in Japanese by three Taiwanese speakers.

The Japanese rule of High Vowel Devoicing (HVD) is regarded as a fast speech rule [3] whereby the high vowels /i/ and /u/ are devoiced at fast rates of speech [4]. To examine the formation/application of this rule by three Taiwanese students at a Japanese university, we examined their production of tokens containing two possible devoicing sites each at two speaker-chosen speeds. Taiwanese students were chosen because it was believed that Taiwanese would have no vowel devoicing due to it being a tonal language, a belief confirmed by inspecting recordings of their productions of Taiwanese. We were looking for evidence of application of the rule of HVD according to its 'textbook' definition-participants should have devoiced more vowels as their rate of speech increased.

Further, it was suspected that as speech rate increased, the duration of voiced vowels should have decreased in some uniform way, in accordance with the Gestural Score model of articulation ([5], [6]) whereby vowel devoicing and vowel deletion in general are seen as a lack of time for articulatory targets to be reached or maintained.

\section{METHODS \& MATERIALS}

\subsection{Participants}

Three Taiwanese learners of Japanese (ID, MK, and TC) and three native speakers of Japanese serving as controls (KS, MT, and TN) participated in the study. All three L2 participants were exchange students studying at a Japanese university, and were paid for their time. Table I details their personal biographical data and their experience with the Japanese language (Japanese experience is the length of time formally studying Japanese).

\begin{tabular}{|c|c|c|c|c|}
\hline $\begin{array}{c}\text { Partici- } \\
\text { pant }\end{array}$ & sex & age & L1-L2/3 & $\begin{array}{c}\text { Japanese } \\
\text { experience }\end{array}$ \\
\hline \hline ID & F & 25 & $\begin{array}{c}\text { Taiwanese } \\
\text { Hakka } \\
\text { Mandarin }\end{array}$ & 3.5 yrs. \\
\hline MK & F & 23 & $\begin{array}{c}\text { Taiwanese } \\
\text { Mandarin }\end{array}$ & 3 yrs. \\
\hline TC & M & 25 & $\begin{array}{c}\text { Taiwanese } \\
\text { Mandarin }\end{array}$ & 4.5 yrs. \\
\hline
\end{tabular}

Table I: L2 participant information.

L1 participants all identify themselves with the Tokyo dialect, of which one distinguishing feature is the rule of HVD. KS, 23 years old, and MT, 25 years old, have lived continuously in Tokyo with very little and moderate conversation foreign language ability, respectively. TN, 34 years old, spent her childhood moving around Japan with her family but spent her high school, college, and middle 20's in Tokyo before moving to the U.S. for seven years.

\subsection{Materials}

Each of following fourteen tokens containing two possible devoicing sites was embedded in the carrier sentence, "Kore wa to iimasu." "This is called (a(n)) .”All tokens were nominals, and although they were not all commonly known, all participants could recognize them through their knowledge of the tokens' Chinese characters. 
- $\quad k i k i$ 'a crisis'

- $k i s h i$ 'a shore'

- kichi 'military base'

- shiki 'conduct'

- $\quad$ shishi 'a lion; Leo'

- chiki 'a friend'

- chichi 'father'

The carrier sentences with embedded tokens were listed in two columns on a single sheet of paper. The sentences were written out in the Japanese syllabary (hiragana); the Chinese character for the token was given at the end of the carrier sentence to disambiguate the semantic meaning and identify the pitch accent.

\subsection{Methods}

Oral and written instructions were given to read the left column of sentences at a slow speed and the right column at a fast speed. Participants read each column in turn five times, using self-determined speech rates, producing 5 repetitions of each token at each speed ( 6 participants $\times 2$ speeds $\times 14$ tokens x 2 vowels $\mathrm{x} 5$ repetitions $=1680$ possible token-internal vowel devoicing sites).

All signals were sampled at $44.1 \mathrm{MHz}$ with a bitwidth of 16 and analyzed using the software Signalyze ${ }^{\mathrm{TM}}$ 3.12. Duration measurements were taken for each token and the F1 of each vowel using waveforms in conjunction with spectrograms.

\section{RESULTS}

\subsection{Speech Rate Effects-Slower vs. Faster Sorting}

Speech rate measurements (as defined by token duration) revealed that none of the participants adequately controlled their rate of speech for all tokens; there was overlap between their slow and fast productions for at least some of the tokens for all speakers. For this reason, the speakers' judgments of slow and fast were not used; instead the ten repetitions of each token were sorted into two equal-size bins, slower and faster, based on token duration.

Figure 1 gives the total number of vowels devoiced at each speed for each speaker using this sorting. For each speaker, the left-hand column represents the number of vowels that were devoiced among all 14 tokens at the slower speech rate; the right-hand column represents the number of vowels that were devoiced among all 14 tokens at the faster speech rate. The bottom portion of each column represents the number of devoiced vowels in the first mora of the tokens (V1), and the upper portion represents the number of devoiced vowels in the second mora of the tokens (V2).

In order to check for significant increases in the number of devoiced vowels at the faster speech rate, two-sample $t$-tests were run on the sorted tokens. Results are reported in Table II.
All tests were pooled right-tailed tests assuming equal variance, except where indicated by the degrees of freedom $(d f)$ in parentheses. In these cases data groups were not pooled; instead the Smith-Satterwaite procedure was used to determine $d f$. (Dashes indicate no difference in number between groups; values reaching significance are marked in bold.)

As can be seen from Table II, only TC among the L2 participants showed any significant speech rate effect (for V1, $t$ $=1.88, p<.05$; for both vowels, $t=2.29, p<.025$ ) Among the L1 participants, only $\mathrm{TN}$ showed a significant speech rate effect, and only for the second vowel $(t=1.59, p<.1)$.

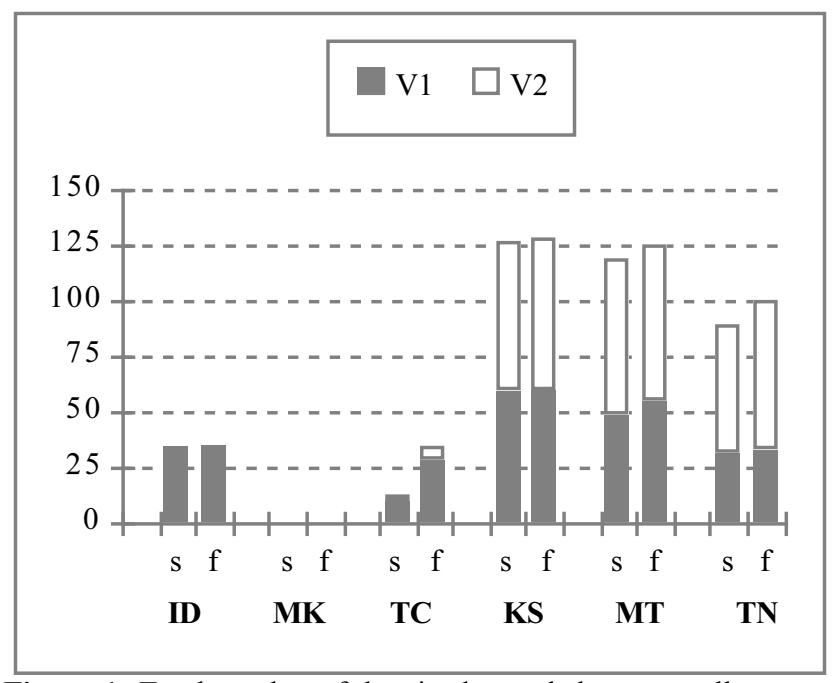

Figure 1: Total number of devoiced vowels by mora, all participants.

\begin{tabular}{|c|c|c|c|}
\hline & V1 & V2 & both vowels \\
\hline ID & $t=.09 ; p>.4$ & $t=1, p<.25$ & $t=0, p>.4$ \\
\hline MK & - & - & - \\
\hline TC & $t=1.88, p<.05$ & $\begin{array}{c}t=.73, p<.25 \\
\text { (21) }\end{array}$ & $\begin{array}{c}t=2.29, p< \\
.025\end{array}$ \\
\hline $\mathbf{K S}$ & $t=0, p \leq 1$ & $\begin{array}{c}t=1.06, p<.25 \\
(21)\end{array}$ & $t=.21, p>.4$ \\
\hline MT & $t=.59, p \leq .4$ & - & $t=.59, p \leq .4$ \\
\hline TN & $t=.18, p>.4$ & $\begin{array}{c}t=1.59, p<.1 \\
(17)\end{array}$ & $\begin{array}{c}t=1.06, p \leq \\
.25\end{array}$ \\
\hline
\end{tabular}

Table II: $t$ and $p$ values for number of vowels devoiced at slower and faster speeds, all participants.

The implications of this lack of speech rate effect for both L2 and L1 speakers will be returned to in the Discussion section.

\subsection{Speech Rate Effects-Gestural Score}

The Gestural score model of articulation ([5], [6]) would seem to predict that an increase in speech rate (i.e. a decrease in token duration) would lead to an increase in vowel reduction/deletion (i.e. a decrease in vowel duration) due to lack of attaining or maintaining the vowel articulations. That is, we would expect to see a positive correlation between token duration and vowel duration. 
To test for such a correlation, regression analysis was performed on the durations of the first vowel vs. the token duration of the productions of the L2 participants. Regression analysis was limited to these tokens due to the scarcity of nonzero values in the L1 participants' productions.

Scattergrams were drawn for each set of ten repetitions of a token by each L2 participant. In cases where there were at least four repetitions that had a non-zero value for V1, a least squares regression lines was calculated. Figure 2 is an example of this.

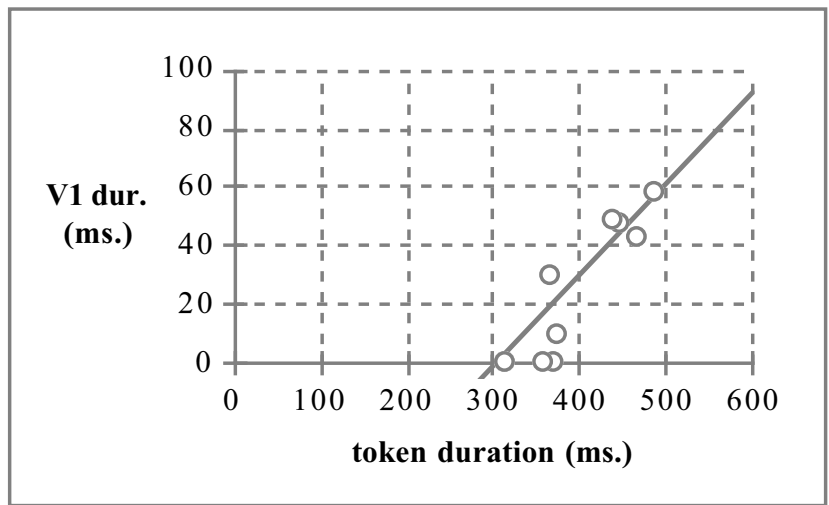

Figure 2: Scattergram of V1 vs. token durations for TC, with least squares regression line, 10 repetitions of kushi. Data points containing a zero value for V1 are not included in the calculation of the regression line.

Of the three L2 participants, TC showed the most consistent positive correlation between token and V1 duration-except for one token, as his speech rate increased, the duration of the V1 decreased. With the exception of one token, ID showed a consistent negative correlation between token and V1 duration-as speech rate increased, the duration of V1 increased. (This reduction was offset by a decrease in the duration of V2.) MK showed no consistent relationship between token and V1 duration.

However, the simple gradient effect suggested by Figure 2 does not hold for other tokens produced by $\mathrm{TC}$ or any other participant. That is, devoicing of these tokens in this sample is not simply a continuous reduction of voicing until the vowel is no longer present. This can be seen in Figure 3, a scattergram of all tokens produced by TC. Two least squares regression lines are fitted to the data points, one calculated from all data points $(\mathrm{m}=.11)$, the other calculated from only those data points that did not include a zero value for V1 $(\mathrm{m}=.04)$. In neither case is it indicated that devoicing of these tokens is the result solely of an increase in speech rate. Rather, due to the wide range of token durations for the devoiced vowels (those on the $\mathrm{x}$ axis), it appears that for TC devoicing is a process that is turned on or off.

\section{DISCUSSION}

\subsection{L2 Participants}

In general, it appears as if the three L2 learners of Japanese are at three different stages of learning the rule of HVD. It appears that only TC among the L2 participants has begun to apply
HVD as a speech-rate dependent rule. However, as indicated by Figure 3, speech rate is not the only conditioning factor.

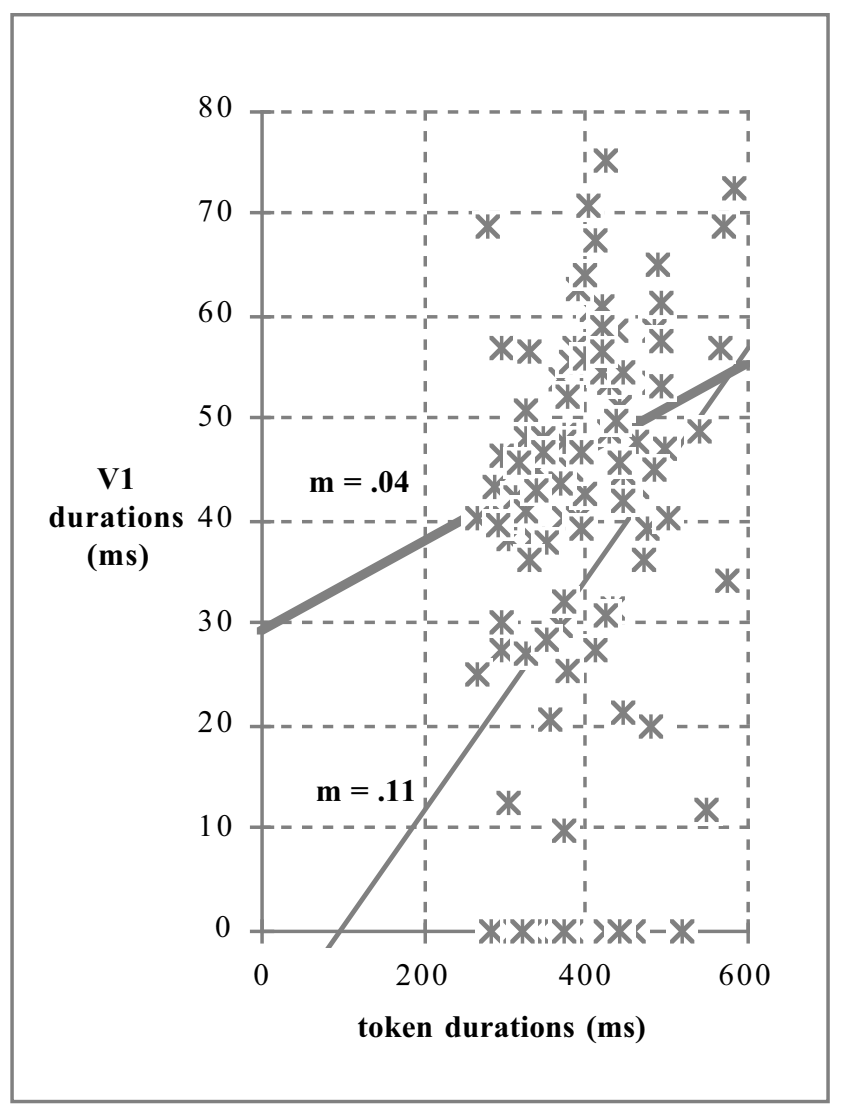

Figure 3: Scattergram of V1 vs. token durations for tokens produced by TC with devoicing in at least one production, including least squares regression line.

ID has evidently begun to be aware of the devoicing of vowels at some level. However, it appears that there is no speech rate effect involved. In addition, the lack of consistent devoicing across tokens indicates that she is not simply learning lexical items that contain devoiced vowels. For ID, there appears to be a rule whose environment is not yet solidified.

Although MK did not devoice any of the vowels contained in the tokens, she did occasionally devoice the sentence-final $/ \mathrm{u} /$ of /iimasu/ 'to say, to call', since the end of this phrase is also a devoicing environment [3]. The other two L2 learners and all three L1 participants regularly devoiced this vowel at both speeds in all of their productions; only MK was variable in this regard. This seems to indicate that $\mathrm{MK}$ is also becoming aware of vowel devoicing at some level, even though she has only begun to apply it sporadically at the end of a phrase. However, it should be noted that it is also possible that MK was suppressing devoicing because of the laboratory setting, and these few final vowels were unintentionally devoiced.

\subsection{L1 Participants}

Perhaps the most outstanding indication to come out of this pilot study was the almost total lack of significant speech rate effect for the L1 participants-outstanding since HVD is 
classically known as a fast speech rule. This same lack of speech rate effect was reported for one participant in another study [7]. Based on the data collected in this study, it appears that the Tokyo dialect rule of HVD may be losing its status as a fast speech rule ([8], [9]), possible becoming a lexicalized rule among the younger generation.

Determining whether this is so will require further study using larger and more varied groups of speakers.

Finally, we will note that fully devoiced vowels (vowels with no F1 present but evidence of an F2 and/or F3) were much more often produced by the L2 participants than by the L1 participants. L1 participants more often produced completely deleted vowels (vowels with no F1, F2 or F3). Examples of the three types of vowels encountered in this pilot study-fully voiced, devoiced, and deleted - can be found on the CD ROM version of the proceedings.

\section{CONCLUSION}

Although the number of repetitions produced for each token are limited, several points were indicated by the data. First, the three L2 learners of Japanese seem to be approaching High Vowel Devoicing in three different manners-for TC, it appears to have begun to become a speech rate dependent rule; for ID, it appears to be a rule that is not speech rate dependent and whose environment is not yet fixed; for MK, it appears to be a rule that is applied only sporadically sentence-finally (although this effect may simply be due to the influence of the laboratory setting).

Other trends indicated were that vowel devoicing is not simply due to gestural overlap for speaker TC (that it, it is not solely a function of speech rate), but instead is indeed a rule that is either applied or not applied.

Further, two types of "devoiced" vowels were found among these samples, those with no F1 present but containing higher formants, and those containing no vowel formants. This seems to indicate that gestural overlap may either involve either a subset of a vowel's articulatory instructions, producing higher formants but not F1 (a devoiced vowel), or may involve all of a vowel's articulatory instructions, producing no formants (a deleted vowel).

Perhaps most intriguing was the lack of significant speech rate effect for the native speakers who served as controls. Of the three, only TN showed a significant speech rate effect, and then only for the second vowel of the tokens. This could be interpreted to mean that HVD is losing its status as a fast speech rule in the Tokyo dialect, becoming instead a lexicalized rule.

Further studies involving larger and more varied groups of both L2 and L1 speakers will indicate if these trends are correct.

\section{REFERENCES}

1. Strange, W. "Phonetics of Second-Language acquisition: Past, present, future." Proc. XIIIth ICPhS 4: 76-83, 1995.

2. Watson, I. "Phonological processing in two languages", in E. Bialystok (ed.) Language Processing in Bilingual Children, Cambridge: CUP, 1991.

3. Hasegawa, N. "Fast speech vs. casual speech", Proc. CLS 15: 126-137, 1979.

4. Vance, T. An Introduction to Japanese Phonology, Albany, NY: SUNY Press, 1989.

5. Browman, C. P., \& Goldstein, L. M. "Gestural specification using dynamically-defined articulatory structures." Journal of Phonetics 18: 299-320, 1990.

6. Browman, C. P., \& Goldstein, L. M. "Articulatory phonology: an overview." Phonetica 49: 155-180, 1992.

7. Kondo, M. "Mechanisms of vowel devoicing in Japanese." In Proc. ICSLP v. 1: 61-64. Yokohama, Japan, 1994.

8. Kuriyagawa, F., and Sawashima, M. "Word accent, devoicing and duration of vowels in Japanese." Annual Bulletin of the Research Institute of Language Processing 23: 85-108, 1989.

9. Maekawa, K. "Hatsuwa Sokudo niyoru yuusei kukan no hendoo." (Speech Rate effects on voicing.) Technical Report SP89-148: 47-53. Densi Joohoo Tsuusin Gakkai, 1990. 